\title{
An iterative method for the computation of nonlinear, wide-angle, pulsed acoustic fields of medical diagnostic transducers
}

\author{
J. Huijssen ${ }^{\text {a) }}$ and M. D. Verweij ${ }^{\text {b) }}$ \\ Laboratory of Electromagnetic Research, Faculty of Electrical Engineering, Mathematics and Computer \\ Science, Delft University of Technology, Mekelweg 4, 2628 CD Delft, The Netherlands
}

(Received 25 June 2009; revised 15 October 2009; accepted 27 October 2009)

\begin{abstract}
The development and optimization of medical ultrasound transducers and imaging modalities require a computational method that accurately predicts the nonlinear acoustic pressure field. A prospective method should provide the wide-angle, pulsed field emitted by an arbitrary planar source distribution and propagating in a three-dimensional, large scale domain holding a nonlinear acoustic medium. In this paper, a method is presented that is free of any assumed wavefield directionality. The nonlinear acoustic wave equation is solved by treating the nonlinear term as a contrast source. This formulation leads to an iterative scheme that involves the repetitive solution of a linear wave problem through Green's function method. It is shown that accurate field predictions may be obtained within a few iterations. Moreover, by employing a dedicated numerical convolution technique, the method allows for a discretization down to two points per wavelength or period of the highest frequency of interest. The performance of the method is evaluated through a number of nonlinear field predictions for pulsed transducers with various geometries. The results demonstrate the directional independence of the method. Moreover, comparison with results from several existing methods shows that the method accurately predicts the nonlinear field for weak to moderate nonlinearity. (C) 2010 Acoustical Society of America. [DOI: 10.1121/1.3268599]
\end{abstract}

PACS number(s): 43.25.Cb, 43.80.Qf [TDM]

Pages: $33-44$

\section{INTRODUCTION}

Current-day medical diagnostic ultrasound scanners benefit from the nonlinear distortion of the acoustic wavefield that arises when the field propagates through human tissue. The imaging modality that is called Tissue Harmonic Imaging (THI) is known to significantly improve the quality of the echographic image by specifically imaging the second harmonic frequency component that arises from the nonlinear propagation in tissue. ${ }^{1,2}$ Recent studies show that the nonlinear distortion effect may be exploited further by employing the third to the fifth harmonic frequency components ${ }^{3,4}$ and by optimizing the ultrasound transducer and the imaging method for the reception of these components. The imaging modality that benefits from the third to the fifth harmonics is called SuperHarmonic Imaging (SHI). The design of a new medical phased array transducer that is optimized for harmonic imaging requires the accurate prediction of the three-dimensional, nonlinear acoustic pressure field generated by such a transducer, even when it is steered over a wide angle. This paper aims at the development of a numerical method that is capable of this. The presented method employs no a priori assumption on the directivity of the wavefield, and it shows an excellent accuracy for the nonlinear fields occurring in medical diagnostic applications.

\footnotetext{
${ }^{a)}$ Present address: Noise and Vibration Research Group, Division PMA, Department of Mechanical Engineering, Catholic University Leuven, Leuven, Belgium.

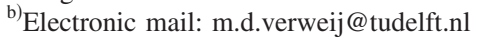

Most currently used three-dimensional, nonlinear, numerical acoustic methods assume forward-wave propagation and are based on an evolution equation that, starting from a source plane, marches the solution from plane to plane in the main direction of propagation. Within this group of methods, a first line of research is based on the KuznetsovZabolotskaya-Khokhlov (KZK) equation. ${ }^{5}$ Because it employs the parabolic or paraxial approximation, the KZK equation is only valid for beams with quasi-planar wavefields. For this reason, the region of validity is generally located not too close to the source and not too far off the main transducer axis. ${ }^{6}$ Currently, a score of numerical implementations is based on the KZK equation and predicts the nonlinear acoustic field of plane source geometries with an arbitrary aperture..$^{7-10}$ A second line of forward-wave methods uses a phenomenological approach and splits each marching step into separate operations that account for the effects of diffraction, absorption, and nonlinearity. ${ }^{11-14}$ The diffraction step is incorporated without using a paraxial approximation. Zemp et al. ${ }^{13}$ employed an efficient angular spectrum approach for this step. Varslot and Taraldsen ${ }^{15}$ developed a forward-wave propagating method for heterogeneous media, and within this context they compared both a parabolic and an angular spectrum approach. For the nonlinear propagation step, all known forward-wave methods employ a plane-wave solution in the time domain or in the frequency domain, thus assuming that the main nonlinear distortion is in the direction of the normal to the computational planes. Although at each diffraction step the nonlinear distortion is spread again, this may not be accurate for wave- 
fields that are strongly focused or that propagate at an angle significantly different from the direction normal to the computational planes. A correction method has been proposed by Christopher and Parker. ${ }^{11}$ Further, Fox et al. ${ }^{16}$ implemented a KZK model with a steered propagation axis, and Christopher ${ }^{17}$ did the same for the phenomenological approach. However, when studying wide-angle phenomena such as grating lobes or propagation through heterogeneous media, nonlinear distortion in all directions needs to be accounted for. A method that handles nonlinear propagation in all forward directions was proposed by Varslot et al., ${ }^{18}$ but this method is limited by its quasilinear approximation.

Apart from the forward-wave methods, a number of fullwave methods have been developed to compute the nonlinear propagation of acoustic fields. ${ }^{19-24}$ Because of the computational effort involved, these methods have been limited to two-dimensional Cartesian or cylindrical implementations. None of these methods has an assumed directionality in the wave propagation or in the nonlinear distortion. An issue with full-wave methods is the fine discretization in space and time that is needed for an accurate computation. In this paper, the discretization is expressed by the number of grid points $D_{\phi}$ per wavelength and per period of a particular field component with temporal (angular) frequency $\phi$. The Nyquist-Shannon sampling theorem for bandlimited signals prescribes a minimum of $D_{\Phi}=2$ for the maximum relevant frequency of interest $\Phi$. Finite difference and finite element methods need at least $D_{\Phi}=10$. Since the number of grid points needed for the computation of a three-dimensional, pulsed field is proportional to $D_{\Phi}^{4}$, a high value of $D_{\Phi}$ makes these methods particularly unfavorable for application to large scale, three-dimensional problems. The best effort in this respect has been made by Wojcik et al., ${ }^{24}$ who presented a pseudospectral method that handles the spatial differentiation in the $k$-space domain. This method requires $D_{\Phi}=4$ in the spatial dimensions, and with a Courant number of 0.2 it employs at least $D_{\Phi}=20$ in the temporal dimension.

The numerical method presented in this paper addresses nonlinear, wide-angle, large scale wave propagation of transient acoustic waves in three dimensions. The main advantages of the method are a nonlinear distortion that is independent of the direction of propagation, and a discretization that is close to $D_{\Phi}=2$ in all dimensions. The former is resolved by an iterative solution of the nonlinear wave problem, and the latter is obtained by an adequate spatiotemporal filtering of the problem. The method will be referred to as the Iterative Nonlinear Contrast Source (INCS) method. The size of the computational domain will be in the order of $100 \times 100 \times 100$ wavelengths and 100 periods at the maximum frequency of interest. In view of the medical diagnostic application, the focus will be on weak to moderate nonlinearity, with special interest in the generation of the second and higher harmonic frequency components. Moreover, local nonlinear effects will be discarded.

This paper starts with the mathematical formulation of the nonlinear wave propagation problem that results in the lossless Westervelt equation. ${ }^{25}$ The nonlinear term in this equation is interpreted as a contrast source, ${ }^{26}$ and the nonlinear wave problem is formally solved with an iterative inte- gral equation scheme. The most significant operation in the repeated steps of the iterative scheme is a convolution of Green's function with the primary and contrast sources. The efficient numerical evaluation of this convolution integral is performed with the Filtered Convolution (FC) method. This method has been described in more detail in a previous paper $^{26}$ that will here be referred to as the FC paper and that will be briefly summarized in this paper. Subsequently, results are presented and discussed for the nonlinear acoustic fields from several types of sources, including medical phased array transducers, and the obtained predictions are compared with results of a number of methods from literature. The paper ends with a discussion of the performance of the method as compared to other methods, and some conclusions.

\section{PROBLEM FORMULATION}

\section{A. Nonlinear wave equation}

Consider a nonlinear fluid that is homogeneous, isotropic, and lossless. The propagation of acoustic waves in this medium may be described by the set of first-order acoustic equations $^{27,28}$

$$
\begin{aligned}
\nabla p+\rho D_{t} \boldsymbol{v} & =\boldsymbol{f}, \\
\nabla \cdot \boldsymbol{v}+\kappa D_{t} p & =q,
\end{aligned}
$$

where $p[\mathrm{~Pa}]$ is the acoustic pressure, $\boldsymbol{v}\left[\mathrm{m} \mathrm{s}^{-1}\right]$ is the particle velocity, and $f\left[\mathrm{~N} \mathrm{~m}^{-3}\right]$ and $q\left[\mathrm{~s}^{-1}\right]$ are the volume densities of the primary sources of force and injection rate, respectively. The medium behavior is accounted for by the density of mass $\rho\left[\mathrm{kg} \mathrm{m}^{-3}\right]$ and the compressibility $\kappa\left[\mathrm{Pa}^{-1}\right]$. Further, $D_{t}=\partial_{t}+\boldsymbol{v} \cdot \boldsymbol{\nabla}$ denotes the total or material time derivative, which is typical for the Eulerian description of wave motion. The second term in $D_{t}$ is an inherent cause of nonlinearity. Another cause for nonlinear behavior is the nonlinearity of the equation of state for the medium. Appendix A shows that the total nonlinear effect is accounted for until the second-order terms in Eqs. (1) and (2) by taking

$$
\begin{aligned}
& \rho=\rho_{0}\left[1+\kappa_{0} p\right], \\
& \kappa=\kappa_{0}\left[1+\kappa_{0}(1-2 \beta) p\right],
\end{aligned}
$$

where $\rho_{0}$ is the ambient density of mass, $\kappa_{0}$ is the ambient compressibility, and $\beta=1+B / 2 A$ is the coefficient of nonlinearity. Appendix B shows that, upon neglection of the local nonlinear effects, Eqs. (1)-(4) lead to the lossless Westervelt equation

$$
c_{0}^{-2} \partial_{t}^{2} p-\nabla^{2} p=S+\frac{\beta}{\rho_{0} c_{0}^{4}} \partial_{t}^{2} p^{2},
$$

where $c_{0}=\left(\rho_{0} \kappa_{0}\right)^{-1 / 2}$ is the small-signal sound speed and $S=\rho_{0} \partial_{t} q-\nabla \cdot f$ represents the primary source.

This paper is concerned with plane source geometries located in the plane $z=0$, e.g., the phased array geometry depicted in Fig. 1. Corresponding to the two terms in $S$, the plane source may be represented as a jump condition in either $\boldsymbol{v}$ or $p$. A velocity jump of magnitude $\Delta V$ is realized by 


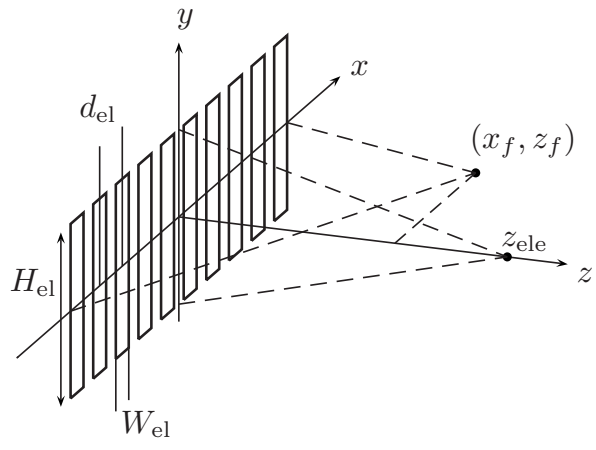

FIG. 1. Plane source geometry for a phased array transducer in the plane $z=0$, employing $N_{\mathrm{el}}$ elements of width $W_{\mathrm{el}}$, height $H_{\mathrm{el}}$, and pitch $d_{\mathrm{el}}$, and lateral focus $\left(x_{f}, z_{f}\right)$ and elevation focus $z_{\text {ele }}$, where $z_{\text {ele }}$ is not necessarily equal to $z_{f}$.

imposing an injection rate acting in an infinitely thin source volume, yielding

$$
S=\rho_{0} \partial_{t}[\Delta V(x, y, t) \delta(z)],
$$

where $\delta(z)$ is the Dirac delta distribution. A pressure jump of magnitude $\Delta P$ is realized by imposing a force acting in an infinitely thin source volume and working perpendicular to its surface, yielding

$$
S=-\partial_{z}[\Delta P(x, y, t) \delta(z)] .
$$

When only one side of the source plane is considered and the medium is homogeneous, these two source descriptions are equivalent to the respective boundary value problems of a transducer with a normal velocity $v_{z}=\Delta V / 2$ in a perfectly rigid baffle, and a transducer with a pressure $p=\Delta P / 2$ in a perfectly compliant baffle.

\section{B. Iterative solution}

The nonlinear acoustic pressure field $p(\boldsymbol{x}, t)$ is interpreted as a summation of a linear field solution $p^{(0)}(\boldsymbol{x}, t)$ and a nonlinear field correction $\delta p(\boldsymbol{x}, t)$. The linear field solution $p^{(0)}$ may be obtained by solving the linearized wave equation

$$
c_{0}^{-2} \partial_{t}^{2} p^{(0)}-\nabla^{2} p^{(0)}=S .
$$

The solution method for the linear wave equation is denoted by the symbolic operator equation $p^{(0)}=\mathcal{L}[S]$. To find $\delta p(\boldsymbol{x}, t)$, the nonlinear term on the right hand side of Eq. (5) is considered as a nonlinear contrast source

$$
S_{\mathrm{nl}}(p)=\frac{\beta}{\rho_{0} c_{0}^{4}} \partial_{t}^{2} p^{2},
$$

which acts in the homogeneous, lossless, and linear background medium associated with Eq. (8). Now, $\delta p$ is related to $S_{\mathrm{nl}}(p)$ as $\delta p=\mathcal{L}\left[S_{\mathrm{nl}}(p)\right]$. If the total nonlinear pressure field $p$ is dominated by $p^{(0)}$, then a good first estimate of $\delta p$ is $\delta p^{(1)}=\mathcal{L}\left[S_{\mathrm{nl}}\left(p^{(0)}\right)\right]$. This yields a first estimate $p^{(1)}=p^{(0)}+\delta p^{(1)}$ of $p$, which may be employed to improve our estimate of $S_{\mathrm{nl}}(p)$. Upon repeated application of these steps, the contrast source formulation enables the computation of a successive estimate $p^{(j)}=p^{(0)}+\delta p^{(j)}$ to $p$, resulting in the Neumann iterative solution ${ }^{29}$

$$
p^{(0)}=\mathcal{L}\left[S^{(0)}\right] \text {, }
$$

$$
\begin{aligned}
& S^{(0)}=S, \\
& p^{(j)}=p^{(0)}+\mathcal{L}\left[S^{(j)}\right], \\
& S^{(j)}=S_{\mathrm{nl}}\left(p^{(j-1)}\right), \quad j \geq 1 .
\end{aligned}
$$

This scheme is the formal equivalent of the approach used by Hoffelner et ll $^{23}$ Moreover, it can be interpreted as a perturbation scheme, ${ }^{5}$ where $p^{(1)}$ is identified as the quasilinear solution. With increasing $j$, this quasilinear solution is iteratively improved toward the full nonlinear wavefield.

One method to compute $\mathcal{L}\left[S^{(j)}\right]$ is the evaluation of the convolution integral ${ }^{30}$

$$
\begin{aligned}
\mathcal{L}\left[S^{(j)}\right](\boldsymbol{x}, t) & =G(\boldsymbol{x}, t) *_{x, t} S^{(j)}(\boldsymbol{x}, t) \\
& =\int_{\mathcal{D}_{T}^{(j)}} \int_{\mathcal{D}_{X}^{(j)}} G\left(\boldsymbol{x}-\boldsymbol{x}^{\prime}, t-t^{\prime}\right) S^{(j)}\left(\boldsymbol{x}^{\prime}, t^{\prime}\right) \mathrm{d} \boldsymbol{x}^{\prime} \mathrm{d} t^{\prime},
\end{aligned}
$$

where $G(\boldsymbol{x}, t)$ is the three-dimensional Green's function

$$
G(\boldsymbol{x}, t)=\frac{\delta\left(t-\|\boldsymbol{x}\| / c_{0}\right)}{4 \pi\|\boldsymbol{x}\|}
$$

of the homogeneous, lossless, and linear background medium. ${ }^{27,31}$ In Eq. $(12), \mathcal{D}_{T}^{(j)}$ and $\mathcal{D}_{X}^{(j)}$ denote the relevant temporal and spatial domains of integration. For $\mathcal{L}\left[S^{(0)}\right]$, the spatial integration is over the support $\mathcal{D}_{X}^{(0)}$ of the plane source $S$ in $z=0$ and involves integrations in $x$ and $y$ only. For the successive estimates $\mathcal{L}\left[S^{(j)}\right]$, the spatial integration is over the three-dimensional support $\mathcal{D}_{X}^{(j)}$ of the contrast source $S^{(j)}$. In principle, the latter support spans the entire space where an acoustic pressure is observed. In practice, however, the domain in which the contrast sources contribute significantly to $p^{(j)}$ is limited, as will be shown in Sec. V A.

The convergence of the scheme may be illustrated for a nonlinear plane-wave problem by comparing the iterations $p^{(j)}$ to an implicit solution $p_{B}$ of the lossless Burgers' equation. $^{32}$ The iterations $p^{(j)}$ are obtained using a onedimensional numerical implementation of Eqs. (10)-(12). ${ }^{28}$ The medium is water $\left(\rho_{0}=998 \mathrm{~kg} \mathrm{~m}^{-3}, c_{0}=1492 \mathrm{~m} \mathrm{~s}^{-1}\right.$, and $\beta=3.52$ ) and the excitation pulse is a harmonic signal with a Gaussian envelope, yielding a pressure pulse with a center frequency of $f_{0}=1 \mathrm{MHz}$, a $2 \sigma$ pulse width of $2.12 \mu \mathrm{s}$, and a maximum surface pressure $P_{0}=500 \mathrm{kPa}$ at the source. Figure 2 shows the frequency spectrum of $p^{(j)}$ at $x=100 \mathrm{~mm}$ for $j=0$ to $j=6$. The convergence of the Neumann iterative solution is very fast. The relative error $\left|\hat{p}^{(j)}(f)-\hat{p}_{B}(f)\right| /\left|\hat{p}_{B}(f)\right|$ has decreased to $0.04 \%$ at the second harmonic $\left(2 f_{0}\right)$ for $j=3$, to $0.12 \%$ at the third harmonic $\left(3 f_{0}\right)$ for $j=4$, and to $0.16 \%$ at the fourth harmonic $\left(4 f_{0}\right)$ for $j=5$. As a rule of thumb, for weak to moderate nonlinear distortion, we may suffice with iteration $j=h+1$ for an accurate prediction of the $h$-th harmonic.

\section{DISCRETIZATION AND NUMERICAL EVALUATION METHOD}

Even in the simplest cases, the convolution integral in Eq. (12) must be discretized and evaluated numerically. As 


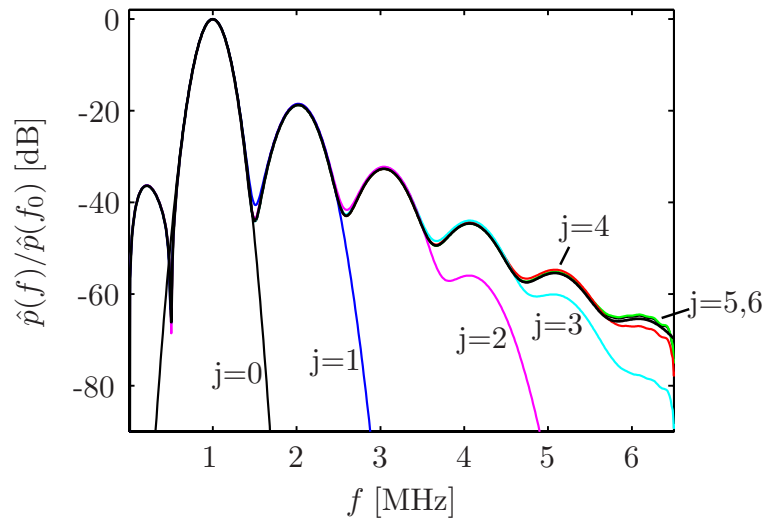

FIG. 2. (Color online) Frequency spectrum of the pressure $p_{B}$ as obtained by the Burgers' equation (thick line) and of the pressure $p^{(j)}$ as obtained by the Neumann iterative solution for $j=0$ to $j=6$ (thin lines). The point of observation is $x=100 \mathrm{~mm}$. Each successive iteration $p^{(j)}$ gives a better estimate for increasingly higher harmonics.

has been discussed in Sec. I, for a full-wave, threedimensional space-time domain method, it is vital to use a discretization that is as coarse as possible, preferably up to the limit of $D_{\Phi}=2$. An additional motivation for this is that with the described iterative solution the pressure field at the full spatiotemporal grid needs to be evaluated and stored in each iteration. Such a coarse discretization may be realized with the FC method. ${ }^{26}$ In the this section the four steps of the FC method will be briefly summarized, and a specific issue in applying this method to the nonlinear wave problem will be discussed.

\section{A. Efficient discretization employing the filtered convolution method}

A straightforward approach to numerically evaluate the convolution integral in Eq. (12) is by approximating it with a convolution sum

$$
\mathcal{L}\left[S^{(j)}\right]_{s, n}=(\Delta x)^{3} \Delta t \sum_{\boldsymbol{r}} \sum_{m} G_{\boldsymbol{s}-\boldsymbol{r}, n-m} S_{\boldsymbol{r}, m}^{(j)},
$$

where $G_{\boldsymbol{r}, m}$ and $S_{\boldsymbol{r}, m}^{(j)}$ are the sampled versions of $G(\boldsymbol{x}, t)$ and $S^{(j)}(\boldsymbol{x}, t)$ on a grid with equidistant points $(\boldsymbol{r} \Delta x, m \Delta t), \Delta x$ and $\Delta t$ being the spatial and temporal step sizes. The resulting values $\mathcal{L}\left[S^{(j)}\right]_{s, n}$ are approximated values of $\mathcal{L}\left[S^{(j)}\right]$ on the grid points $(s \Delta x, n \Delta t)$, and the error strongly depends on the chosen step sizes. In order to attain a coarse discretization, it is assumed that a maximum angular frequency of interest $\Phi$ may be chosen in such a way that all components of interest in the pressure field have a temporal angular frequency $|\omega| \leq \Phi$ and a spatial angular frequency $|\boldsymbol{k}|=\left(k_{x}^{2}+k_{y}^{2}+k_{z}^{2}\right)^{1 / 2} \leq \Phi / c_{0}$. The first steps of the FC method are to filter and window $G(\boldsymbol{x}, t)$ and $S^{(j)}(\boldsymbol{x}, t)$ in all spatiotemporal dimensions before these functions are sampled. This means that $G(\boldsymbol{x}, t)$ and $S^{(j)}(\boldsymbol{x}, t)$ are subjected to an ideal low-pass filter with an angular cutoff frequency $\Omega=\pi / \Delta t$ in the temporal domain and ideal, spherical low-pass filter with an angular cutoff frequency $K=\pi / \Delta x$ in the spatial domain. This avoids the aliasing error that would otherwise be caused during the sampling operation by the frequency components $|\omega|>\Omega$ and $\left\{\left|k_{x}\right|,\left|k_{y}\right|,\left|k_{z}\right|\right\}>K$. Because of the assumption on the frequency content of interest, $\Omega$ may be taken as small as $\Phi$ and $K$ as small as $\Phi / c_{0}$. The resulting step sizes may therefore be as large as $\Delta t=\pi / \Phi$ and $\Delta x=c_{0} \pi / \Phi$, resulting in a discretization of $D_{\Phi}=2$. To limit the number of points in each dimension and thus enable the evaluation of a limited convolution sum, a rectangular window is applied to $G(\boldsymbol{x}, t)$ and $S^{(j)}(\boldsymbol{x}, t)$ in all spatiotemporal dimensions. After the filtering and windowing operations, the second step of the FC method is to sample $G(\boldsymbol{x}, t)$ and $S^{(j)}(\boldsymbol{x}, t)$ at a coarse discretization of $D_{\Phi}=2$. The last two steps of the FC method involve the evaluation of the resulting convolution sum in Eq. (14) with a Fast Fourier Transform (FFT) method. To achieve this, in step $3, G_{\boldsymbol{r}, m}$ and $S_{\boldsymbol{r}, m}^{(j)}$ are transformed with fourdimensional FFTs, and in step 4 the obtained results are multiplied and transformed back to the original domain with an inverse FFT to yield $\mathcal{L}\left[S^{(j)}\right]_{s, n}$.

\section{B. Spatiotemporal filtering of the nonlinear contrast source}

In the FC paper, ${ }^{26}$ the spatiotemporally filtered and windowed version of the three-dimensional Green's function in Eq. (13) is obtained analytically. In the same paper, the spatiotemporal filtering and windowing of the primary source, as well as the filtering and windowing of a general contrast source, are thoroughly discussed. In this subsection, several aspects are exemplified that are specific to the spatiotemporal filtering of the nonlinear contrast source.

For the evaluation of the contrast source $S^{(j)}$ the pressure field $p^{(j-1)}$ is used. It is observed that $p^{(j-1)}$ is bandlimited in all spatiotemporal dimensions if it has been obtained with the FC method. At first sight it seems that the squaring of $p^{(j-1)}$ generates a result with a spatiotemporal bandwidth that may exceed both the angular cutoff frequency $\Omega$ and cutoff wavenumber $K=\Omega / c_{0}$, and filtering must be applied in all dimensions to prevent aliasing. However, since we may assume that $p^{(j-1)}$ predominantly consists of propagating waves for which the spatial and temporal behaviors are related by $|k|=\omega / c_{0}$, the numerical evaluation may be considerably simplified by omitting the filtering in the spatial dimensions and applying the filter only in the temporal dimension. This is shown by first studying the behavior of the nonlinear contrast source $S_{\mathrm{nl}}(p)$ for a pressure field $p(\boldsymbol{x}, t)$ that consists of two steady-state, two-dimensional, plane waves traveling in the plane $y=0$, having frequencies $\omega_{1}$ and $\omega_{2}$ with $0 \leq \omega_{2} \leq \omega_{1} \leq \Omega$, and crossing each other at an angle $0 \leq \alpha \leq \pi$,

$$
\begin{aligned}
p(x, t)= & I_{\omega_{1}}+I_{\omega_{2}} \\
= & \sin \left[\omega_{1} t-\frac{\omega_{1}}{c_{0}} s_{\alpha} x-\frac{\omega_{1}}{c_{0}} c_{\alpha} z\right] \\
& +\sin \left[\omega_{2} t+\frac{\omega_{2}}{c_{0}} s_{\alpha} x-\frac{\omega_{2}}{c_{0}} c_{\alpha} z\right],
\end{aligned}
$$

where $s_{\alpha}=\sin (\alpha / 2)$ and $c_{\alpha}=\cos (\alpha / 2)$. The square of $p(\boldsymbol{x}, t)$ that occurs in $S_{\mathrm{nl}}(p)$ consists of five terms

$$
p^{2}(\boldsymbol{x}, t)=I_{0}+I_{2 \omega_{1}}+I_{2 \omega_{2}}+I_{\omega_{1}+\omega_{2}}+I_{\omega_{1}-\omega_{2}},
$$

where 


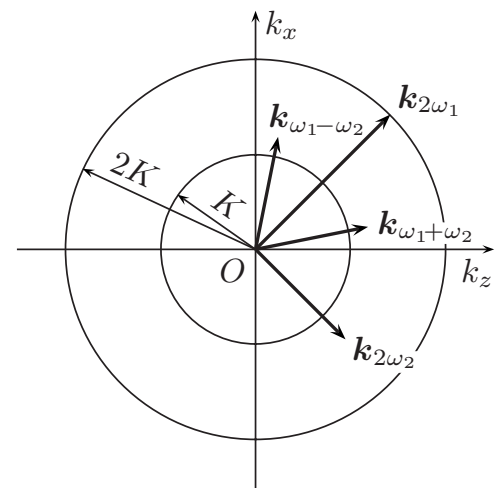

FIG. 3. Location of the wavevectors $k$ associated with the terms $I_{2 \omega_{1}}, I_{2 \omega_{2}}$, $I_{\omega_{1}+\omega_{2}}$, and $I_{\omega_{1}-\omega_{2}}$ for the cases $\omega_{1}=\Omega, \omega_{2}=(2 / 3) \Omega$, and $\alpha=90^{\circ}$.

$$
I_{0}=1
$$

$$
\begin{aligned}
& I_{2 \omega_{1}}=-\frac{1}{2} \cos \left[2 \omega_{1} t-\frac{2 \omega_{1}}{c_{0}} s_{\alpha} x-\frac{2 \omega_{1}}{c_{0}} c_{\alpha} z\right] \\
& I_{2 \omega_{2}}=-\frac{1}{2} \cos \left[2 \omega_{2} t+\frac{2 \omega_{2}}{c_{0}} s_{\alpha} x-\frac{2 \omega_{2}}{c_{0}} c_{\alpha} z\right] \\
& I_{\omega_{1}+\omega_{2}}=-\cos \left[\left(\omega_{1}+\omega_{2}\right) t-\frac{\omega_{1}-\omega_{2}}{c_{0}} s_{\alpha} x-\frac{\omega_{1}+\omega_{2}}{c_{0}} c_{\alpha} z\right]
\end{aligned}
$$

$$
I_{\omega_{1}-\omega_{2}}=\cos \left[\left(\omega_{1}-\omega_{2}\right) t-\frac{\omega_{1}+\omega_{2}}{c_{0}} s_{\alpha} x-\frac{\omega_{1}-\omega_{2}}{c_{0}} c_{\alpha} z\right]
$$

Due to the second-order time derivative in Eq. (9), the term $I_{0}$ does not yield a contribution to $S_{\mathrm{nl}}(p)$. The remaining terms are of the form $I_{\omega}=a \cos \left(\omega t-k_{x} x-k_{z} z\right)$, in which $k_{x}$ and $k_{z}$ may be considered as the components of a wavevector that depends on the particular values of $\omega_{1}, \omega_{2}$, and $\alpha$. Figure 3 shows the wavevectors for a typical situation. The temporal filtering removes each term $I_{\omega}$ with $\omega>\Omega$. Inspection of Eqs. (18)-(20) shows that the terms $I_{2 \omega_{1}}, I_{2 \omega_{2}}$, and $I_{\omega_{1}+\omega_{2}}$ that survive the temporal filtering also have $k_{x} \leq K$ and $k_{z} \leq K$. This implies that the terms associated with the wavevectors $\boldsymbol{k}_{2 \omega_{1}}$, $\boldsymbol{k}_{2 \omega_{2}}$, and $\boldsymbol{k}_{\omega_{1}+\omega_{2}}$ in Fig. 3 are eliminated by the temporal filtering. On the other hand, the temporal filtering will never remove a term $I_{\omega_{1}-\omega_{2}}$ because it always occurs that $\left(\omega_{1}-\omega_{2}\right) \leq \Omega$. Inspection of Eq. (21) reveals that although for this term $k_{z} \leq K$, there are circumstances under which $k_{x}=\left(\omega_{1}+\omega_{2}\right) s_{\alpha} / c_{0}>K$, and these are not dealt with by the temporal filtering. This implies that the term with the wavevector $\boldsymbol{k}_{\omega_{1}-\omega_{2}}$ in Fig. 3 remains present if no spatial filtering is applied. The issue is now to determine for which values of $\omega_{1}=\gamma \Omega(0 \leq \gamma \leq 1), \omega_{2}=\eta \omega_{1}(0 \leq \eta \leq 1)$, and $\alpha$ the term $I_{\omega_{1}-\omega_{2}}$ will still possess a $k_{x} \leq K$ and, consequently, the spatial filtering of the contrast source may still be skipped. It turns out that this is the case if

$$
(1+\eta) \gamma \sin \left(\frac{\alpha}{2}\right) \leq 1
$$

This inequality always holds if $\gamma \leq 1 / 2$, i.e., for frequencies $\omega_{2} \leq \omega_{1} \leq \Omega / 2$, and independently if $\alpha \leq \pi / 3$, i.e., for a crossing angle smaller than $60^{\circ}$. For other values of $\gamma$ and $\alpha$, only specific ranges of $\eta$ yield a spatial frequency larger than $K$. In conclusion, for the case of two crossing plane waves, the temporal filtering with a cutoff frequency $\Omega$ implies spatial filtering with a cutoff frequency less than or equal to $K=\Omega / c_{0}$ for all frequencies smaller than $\Omega / 2$ and for all crossing angles smaller than $60^{\circ}$.

The considered case of two crossing plane waves can be generalized to a realistic three-dimensional situation where the nonlinear pressure field $p$ consists of a continuum of plane waves, all with temporal frequencies below $\Omega$. In this situation, the pressure field in points that are close to the source shows a different characteristic than the pressure field in points that are some distance away from the source. For points close to the source, the cumulative nature of nonlinear propagation results in a pressure field in which the higher harmonic components are virtually absent, and the field only consists of the fundamental frequency components in a small frequency band around $f_{0}$. In any computation involving nonlinear fields, the maximum frequency of interest $F$ $=\Omega / 2 \pi$ is chosen such that at least the second harmonic is incorporated, i.e., $F \geq 2 f_{0}$. As a consequence, $\gamma \leq 1 / 2$ for the temporal frequencies involved with the field near the source, and the spatial filtering will be superfluous here. For points at some distance from the source, the constituents of $p$ will travel more or less parallel to each other and cross each other under an angle far less than $60^{\circ}$. This causes that at some distance from the source $\alpha<\pi / 3$ and the spatial filtering may safely be omitted as well.

\section{IMPLEMENTATION}

The method has been implemented in FORTRAN on a single processor system as well as on a multiprocessor parallel system with distributed memory. The FFTs are performed with routines from the FFTW library. ${ }^{33}$ The evaluation of the partial derivatives in the primary and contrast sources utilizes either a high order finite difference scheme or a spectral derivative. ${ }^{34}$ The structure of the parallel program may be found in the FC paper. ${ }^{26}$ Although with the FC method the discretization occurs at the theoretical limit of two points per wavelength, a number of additional computational strategies may be invoked to realize a further reduction in memory and computation time. First, symmetrical source geometries allow the reduction in the spatial computational domain by only computing the fields for $x \geq 0$ and/or $y \geq 0$, and deriving from them the contrast sources at $x<0$ and/or $y<0$. Second, reduction in the temporal dimension may be obtained by using a periodical time axis, which is achieved by omitting the temporal windowing operation on Green's function. Third, the convolution sum may be split up and evaluated in blocks, thus reducing the demand in core memory. Finally, instead of only using fixed rectangular domains, a co-moving time frame along the $z$-dimension may be employed, or a domain in the shape of a parallellogram in either the 
$(x, z)$-plane or the $(y, z)$-plane may be used in case of steered beams or grating lobes under a large angle with the $z$-axis. This results in a smaller time window and/or spatial domain, especially for large observation angles. The skew domain may be wrapped in such a way that a rectangular domain results, which may then be efficiently dealt with by an FFT method. ${ }^{35}$

\section{NUMERICAL RESULTS}

In this section results are presented that are obtained by the INCS method for the nonlinear field of a point source, an unfocused cylindrical piston transducer with radius $R$, a rectangular transducer with width $W$ and height $H$, and a focused and steered phased array transducer as shown in Fig. 1. All plane source representations will employ a pressure jump condition. The source geometry is included by a geometry factor $0 \leq A(x, y) \leq 1$. The pulse that will be used is a harmonic signal with a Gaussian envelope, resulting in a pressure jump

$$
\Delta P(x, y, t)=2 P_{0} A(x, y) \exp \left[-\left(2 t / t_{w}\right)^{2}\right] \sin \left(2 \pi f_{0} t\right),
$$

where $P_{0}$ is the maximum surface pressure of the source, $f_{0}$ is the center frequency, and $t_{w}$ is the pulse width. The position-dependent delay of the time signature needed for the focusing of the phased array is accounted for in the temporal Fourier domain by using a geometry factor

$$
\hat{A}(x, y, \omega)=A(x, y) \exp \left[-j \omega t_{d}(x, y)\right],
$$

where $t_{d}(x, y)$ is the delay time. The medium is water $\left(\rho_{0}=998 \mathrm{~kg} \mathrm{~m}^{-3}, c_{0}=1492 \mathrm{~m} \mathrm{~s}^{-1}\right.$, and $\left.\beta=3.52\right)$, and attenuation is considered negligible.

\section{A. Domain of the contrast source}

The first results concern the spatial extent of the contrast sources that contribute significantly to the nonlinear field solution. As the nonlinear distortion is formed cumulatively along the propagation path up to a certain point of observation, it may be assumed beforehand that those nonlinear contrast sources that lie in the region in between the primary source and the observation point will mainly contribute to the nonlinear distortion, and that the influence of other contrast sources can be safely ignored. This assumption may be investigated by using the INCS method to compute the nonlinear field correction due to the entire contrast source, and to compare this with the nonlinear field correction obtained with a contrast source that is partially masked. The primary source is a rectangular piston with dimensions $W \times H$ $=10 \mathrm{~mm} \times 10 \mathrm{~mm}$, causing a pressure jump. The source emits a harmonic signal with a frequency of $f_{0}=1 \mathrm{MHz}$ and a Gaussian envelope with a pulse width of 6 cycles, i.e., $t_{w}$ $=3 / f_{0}$, and a maximum surface pressure of $P_{0}=500 \mathrm{kPa}$. The pressure field is obtained in a domain of interest with a spatial size of $15 \mathrm{~mm} \times 15 \mathrm{~mm} \times 52 \mathrm{~mm}$ and a co-moving temporal window of $22 \mu \mathrm{s}$. The maximum frequency of interest is $F=3 \mathrm{MHz}$, the discretization is performed at $D_{F}$ $=2$, and the linear field solution $p^{(0)}$ and the first nonlinear field correction $\delta p^{(1)}$ are obtained. The profile of $p^{(0)}$ is shown in Fig. 4(a), and the profile of $\delta p^{(1)}$, employing the
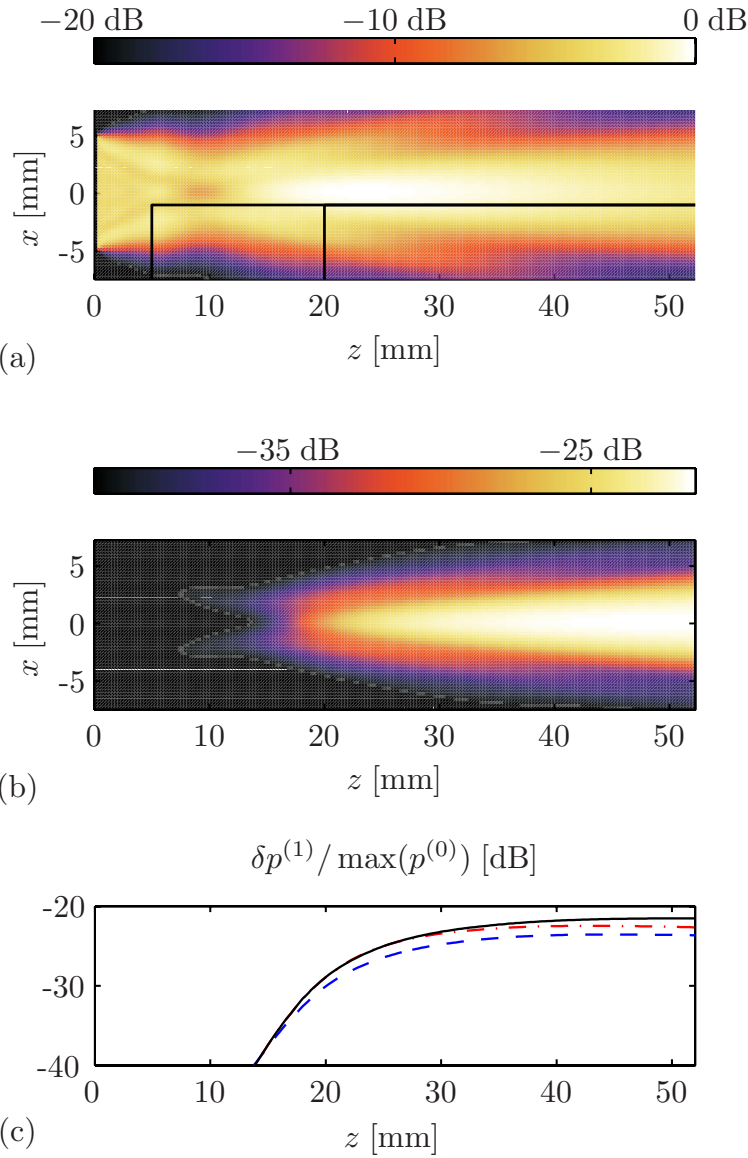

FIG. 4. (Color online) Profiles for the rectangular piston source. (a) The linear field solution $p^{(0)}$ in the plane $y=0 \mathrm{~mm}$, together with the masking domains. (b) The nonlinear field correction $\delta p^{(1)}$ in the plane $y=0 \mathrm{~mm}$, for the unmasked case. (c) $\delta p^{(1)}$ on the $z$-axis, for the unmasked case (solid), for a masking domain starting at $z=5 \mathrm{~mm}$ (dashed) and for a masking domain starting at $z=20 \mathrm{~mm}$ (dashed-dotted). The $0 \mathrm{~dB}$ level corresponds to 870 $\mathrm{kPa}$.

entire region of interest as the contrast source domain, is depicted in Figs. 4(b) and 4(c). If the contrast source domain is extended to a size of $30 \mathrm{~mm} \times 30 \mathrm{~mm} \times 104 \mathrm{~mm}$, then $\delta p^{(1)}$ does not change noticeably, which indicates that the original domain was indeed sufficiently large to compute the profiles in Figs. 4(b) and 4(c).

Next, the contrast source is masked, i.e., put to zero, in a region $X \times Z=[-7.5 \mathrm{~mm},-1 \mathrm{~mm}] \times[z, 52 \mathrm{~mm}]$ with $z$ $=\{5,20\} \mathrm{mm}$, as depicted in Fig. 4(a). In this case an error $p_{z}^{(1)}-p^{(1)}=\delta p_{z}^{(1)}-\delta p^{(1)}$ in the first nonlinear field estimate shows up, where the subscript $z$ denotes the specific masking domain. From Fig. 4(c) it becomes clear that the effect of this masking in the axial profile mainly manifests itself as an underestimation of the nonlinear field correction. This effect is larger for a masking domain starting closer to the primary source. The profiles of the errors $\delta p_{z}^{(1)}-\delta p^{(1)}$ are shown in Fig. 5. From these figures it may be observed that the less the masking blocks the view from an observation point to the primary source, the lower the error in the nonlinear field correction becomes. This confirms that, at least for a plane primary source, the contrast sources that contribute significantly to the nonlinear field in a certain observation point indeed lie in the region in between the observation point and 

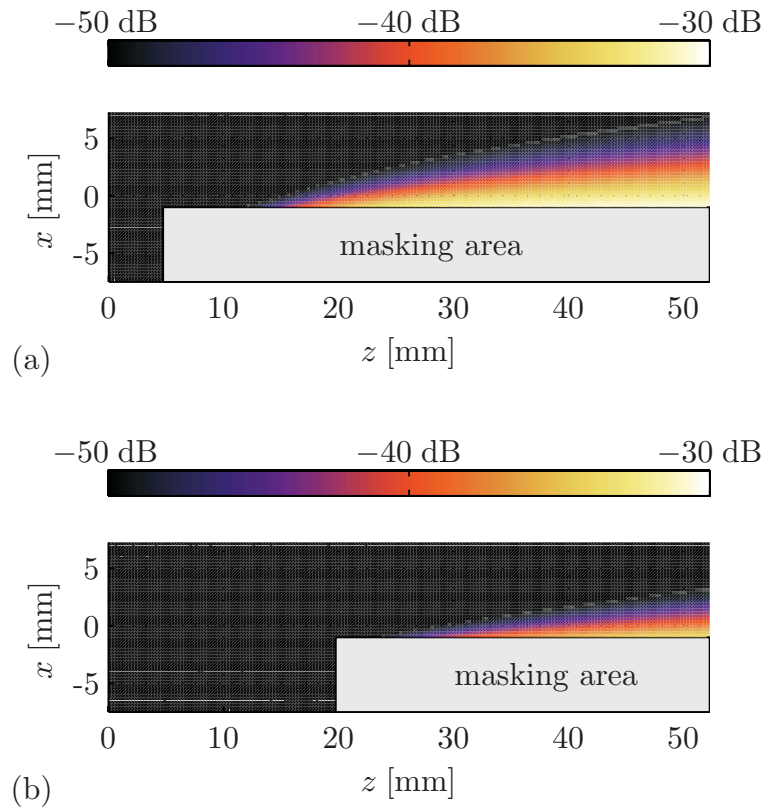

FIG. 5. (Color online) Profiles of the error $\delta p_{z}^{(1)}-\delta p^{(1)}$ in the nonlinear field corrections caused by the masking of the contrast source domains. The plots show the profiles in the plane $y=0 \mathrm{~mm}$ for the masking domains starting at (a) $z=5 \mathrm{~mm}$ and (b) $z=20 \mathrm{~mm}$. The $0 \mathrm{~dB}$ level corresponds to $870 \mathrm{kPa}$.

the primary source. This knowledge can be used to reduce the support of the contrast source and thus to reduce the numerical effort needed for the iterations of the INCS method.

\section{B. Non-directionality of the nonlinear distortion}

An important benefit of the INCS method is the directional independence of the linear and nonlinear field solutions. This may be demonstrated by computing the nonlinear pressure field from a omnidirectional point source located at $\boldsymbol{x}=\mathbf{0}$, defined as

$$
S=S_{0} S(t) \delta(x) .
$$

In the linear case, the field solution for the point source is found by substituting Eq. (25) and the three-dimensional Green's function of Eq. (13) in Eq. (12). This yields

$$
p_{L}(\boldsymbol{x}, t)=\frac{S_{0} S\left(t-\|\boldsymbol{x}\| / c_{0}\right)}{4 \pi\|\boldsymbol{x}\|}, \quad \boldsymbol{x} \neq 0 .
$$

The signature $S(t)$ is a harmonic signal with a unit amplitude and a Gaussian envelope with $f_{0}=1 \mathrm{MHz}$ and $t_{w}=3 / f_{0}$. The amplitude factor $S_{0}$ equals $2 \pi \times 10^{3} \mathrm{~N} \mathrm{~m}^{-1}$, resulting in a maximum linear pressure of $p_{L}=500 \mathrm{kPa}=p_{1 \mathrm{~mm}}$ at $\|\boldsymbol{x}\|=1 \mathrm{~mm}$. In the nonlinear case, the domain of interest is centered at the origin and has a size of $8.6 \mathrm{~mm} \times 8.6 \mathrm{~mm}$ $\times 8.6 \mathrm{~mm}$, and a co-moving temporal window of $12 \mu \mathrm{s}$ is used. The maximum frequency of interest is $F=5 \mathrm{MHz}$, the discretization is performed at $D_{F}=2$, and the iterative scheme is run up to $j=3$. Figure 6(a) shows the profile for the linear field $p^{(0)}$ in the plane $y=0$, and Fig. 6(b) shows the profile on the $z$-axis, together with the profile of the linear field solution $p_{L}$ from Eq. (26). As observed from the latter figure, the INCS method reproduces the linear field profile very accurately, except for a very small region around the point source.
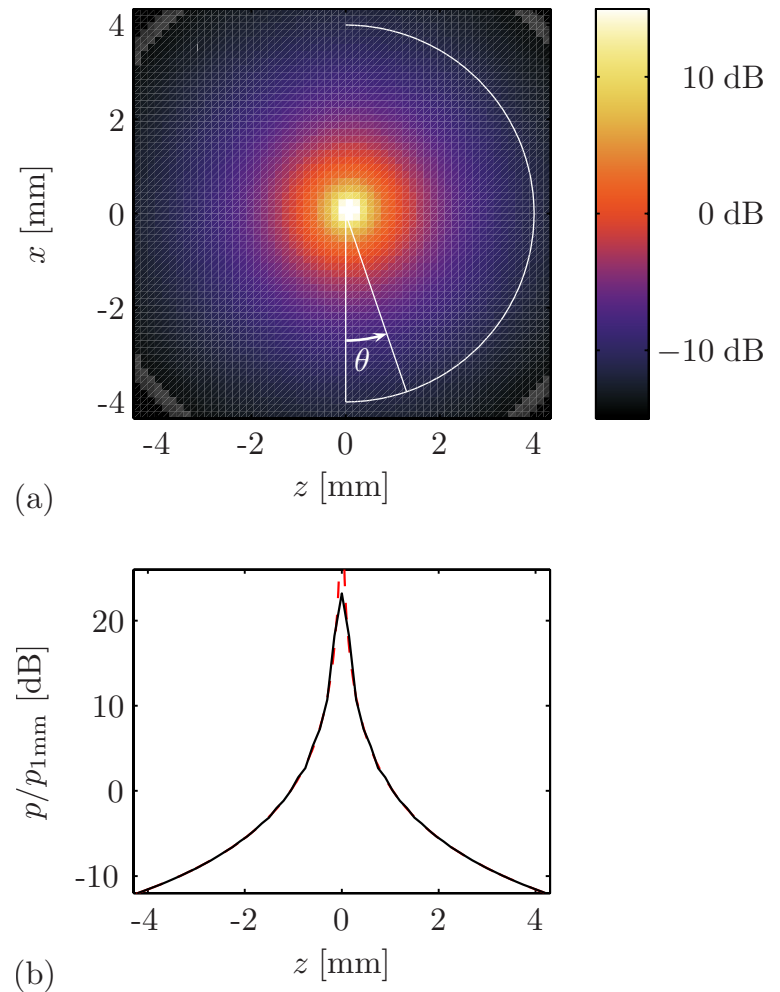

FIG. 6. (Color online) Profile of $p^{(0)}$ for the point source. (a) In the plane $y=0$, together with the half-circle used in Fig. 7. (b) On the $z$-axis, as obtained from the INCS method (solid) and from the analytical solution in Eq. (26) (dashed). The $0 \mathrm{~dB}$ level corresponds to $500 \mathrm{kPa}$.

The directional independence of the INCS method is demonstrated by the profile of the linear field solution $p^{(0)}$ and the profile of the nonlinear field correction $\delta p^{(3)}=p^{(3)}$ $-p^{(0)}$ on the half-circle with radius $\|x\|=4 \mathrm{~mm}$ that is indicated in Fig. 6(a). Figure 7 shows the relative variation of the mentioned radial profiles around their mean levels, versus

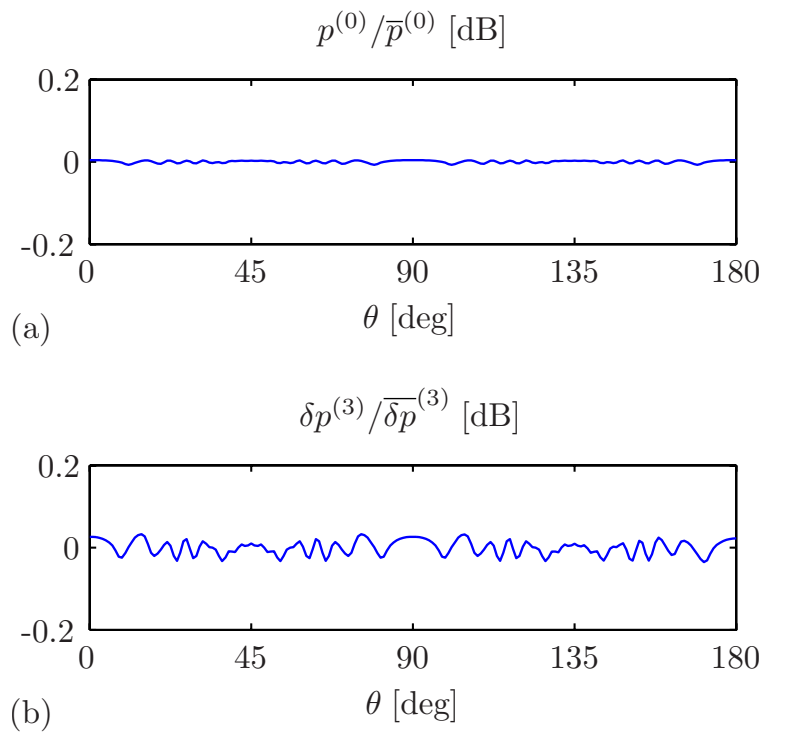

FIG. 7. (Color online) Radial profile levels at a semi-circle with radius $\|\boldsymbol{x}\|=4 \mathrm{~mm}$ as indicated in Fig. 6(a), versus angle $\theta$. (a) For the linear field solution $p^{(0)}$. (b) For the nonlinear field correction $\delta p^{(3)}$. The mean levels of the linear field profile and the nonlinear field correction profile at that radius are $\bar{p}^{(0)}=123 \mathrm{kPa}$ and $\overline{\delta p}(3)=0.97 \mathrm{kPa}$. 
$\hat{p}\left(f_{h}\right) / \max \left[\hat{p}\left(f_{0}\right)\right][\mathrm{dB}]$
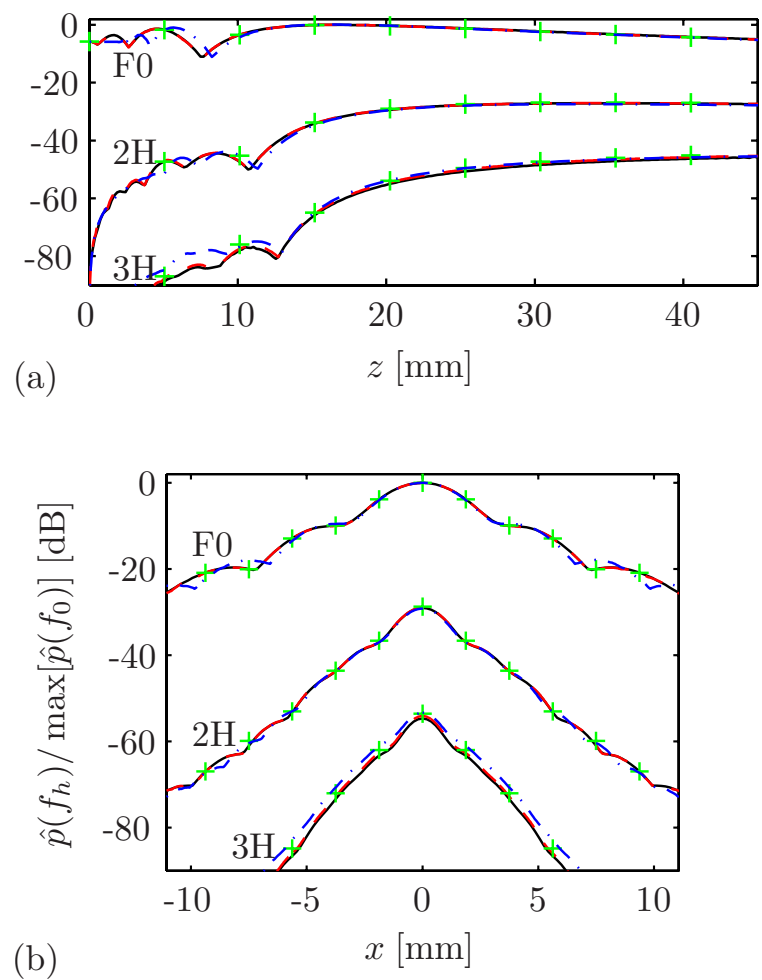

FIG. 8. (Color online) Spectral profiles for the cylindrical source as obtained by the INCS method (solid), the FD-WV method (dashed), the AS-NLP method (+), and the KZK method (dashed-dotted). (a) Axial spectral profiles. (b) Lateral spectral profiles at $z=20 \mathrm{~mm}$. The labels $F 0,2 H$, and $3 H$, respectively, indicate the fundamental, second harmonic, and third harmonic. The $0 \mathrm{~dB}$ level corresponds to $983 \mathrm{kPa}$.

the angle $\theta$. It may be observed that the relative directional variation is less than $0.05 \mathrm{~dB}$. This fact confirms that the INCS method is virtually directionally independent.

\section{Comparison with other methods for the field of a cylindrical piston transducer}

To verify the accuracy of the INCS method, the results for an unfocused cylindrical piston transducer are compared with those of the following three existing methods: The Finite Difference Westervelt (FD-WV) method, ${ }^{22,36}$ which is an FD approximation of the Westervelt equation for axisymmetric configurations, the Angular Spectrum NLP (AS-NLP) method, ${ }^{13}$ which is a marching step scheme based on the angular spectrum approach, and a method based on the KZK equation. ${ }^{37}$ The employed transducer has a radius $R=5 \mathrm{~mm}$, and is excited with a source pulse as used before with $f_{0}=1 \mathrm{MHz}, t_{w}=3 / f_{0}$, and $P_{0}=500 \mathrm{kPa}$. The three existing methods are run with parameter settings that should result in an accurate field prediction. The INCS method employs a domain of interest with a spatial size of $17 \mathrm{~mm} \times 11 \mathrm{~mm}$ $\times 45 \mathrm{~mm}$ and a co-moving temporal window of $39 \mu \mathrm{s}$, a maximum frequency of interest of $F=4 \mathrm{MHz}$ with a discretization at $D_{F}=2$, and iteration $j=4$ is investigated. Figure 8 shows the spectral profiles of the fundamental and the second and third harmonics on the axis of the transducer, and in a radial direction at $z=20 \mathrm{~mm}$. The profiles of the INCS method show a good agreement with those of the other meth- ods. The agreement is best for the FD-WV method, with a difference of at most $0.3 \mathrm{~dB}$ for the fundamental and second harmonic and $0.9 \mathrm{~dB}$ for the third harmonic. With the ASNLP method, the difference is at most $1.0 \mathrm{~dB}$ for the fundamental and second harmonic, and $2.0 \mathrm{~dB}$ for the third harmonic. The difference with the KZK code within the paraxial region, which is the region not too close to the source and up to $\pm 16^{\circ}$ off the transducer axis, is at most $1.0 \mathrm{~dB}$ for the fundamental and second harmonic, and $3.0 \mathrm{~dB}$ for the third harmonic. For locations near the transducer axis, these comparisons confirm that the INCS method provides an accurate prediction of the fundamental and the higher harmonics.

\section{Comparison with the AS-NLP method for the field of a phased array transducer}

In a second accuracy test, the nonlinear field profiles are compared as predicted by the INCS method and by the ASNLP method for a 48 element phased array transducer with element parameters $W_{\mathrm{el}} \times H_{\mathrm{el}}=0.21 \mathrm{~mm} \times 12 \mathrm{~mm}$, and $d_{\mathrm{el}}=0.5 \mathrm{~mm}$. The array is excited with a source pulse as used before with $f_{0}=1 \mathrm{MHz}, t_{w}=3 / f_{0}$ and $P_{0}=250 \mathrm{kPa}$. The nonlinear field solution as obtained with the INCS method is evaluated in a domain of interest with a spatial size of $30 \mathrm{~mm} \times 18 \mathrm{~mm} \times 72 \mathrm{~mm}$ and a co-moving temporal window of $36 \mu \mathrm{s}$. The maximum frequency of interest is $F=4 \mathrm{MHz}$, the discretization is performed at $D_{F}=2$, and the iteration $j=4$ is investigated.

First we consider an unsteered beam. In this case the array is focused at $(0 \mathrm{~mm}, 57 \mathrm{~mm})$, with $z_{\text {ele }}=z_{f}$. Figure 9 shows the spectral profiles of the fundamental and the second and third harmonics, as obtained with the INCS method. In Fig. 10 the axial spectral profiles and the lateral spectral profiles for $y=0 \mathrm{~mm}$ and $z=58 \mathrm{~mm}$ are shown, as obtained with the INCS method and the AS-NLP method. These figures show an even better resemblance between the spectral profiles of the INCS method and the AS-NLP method than for the unfocused cylindrical source.

From the directional independence observed in Sec. $\mathrm{V} \mathrm{B}$, it is assumed that the INCS method will equally well predict the spectral profiles of the unsteered beam as considered above, and of a beam steered $45^{\circ}$ off the transducer axis as considered next. The focus in this case is $\left(x_{f}, z_{f}\right)=$ (40 mm, $40 \mathrm{~mm}$ ), with $z_{\text {ele }}=z_{f}$, giving the same focal distance as in the unsteered case. The domain of interest is parallellogram-shaped in the $x z$-plane, conforming the beam angle, and this skew domain has a size of $30 \mathrm{~mm}$ $\times 18 \mathrm{~mm} \times 53 \mathrm{~mm}$. In Fig. 11, the fundamental, the second harmonic, and the third harmonic spectral profiles in the plane $y=0 \mathrm{~mm}$ are shown, as obtained with the INCS method with the same discretization and number of iterations as in the unsteered situation. Figure 12 shows the lateral spectral profiles for $y=0 \mathrm{~mm}$ and $z=40 \mathrm{~mm}$, as obtained by the INCS method and the AS-NLP method. For the latter, similar parameter settings as for the unsteered case are utilized. The direction perpendicular to the computational planes is kept in the direction of the $z$-axis, and in the current case it is therefore not directed along the steered beam axis. For the fundamental component, a perfect agreement be- 


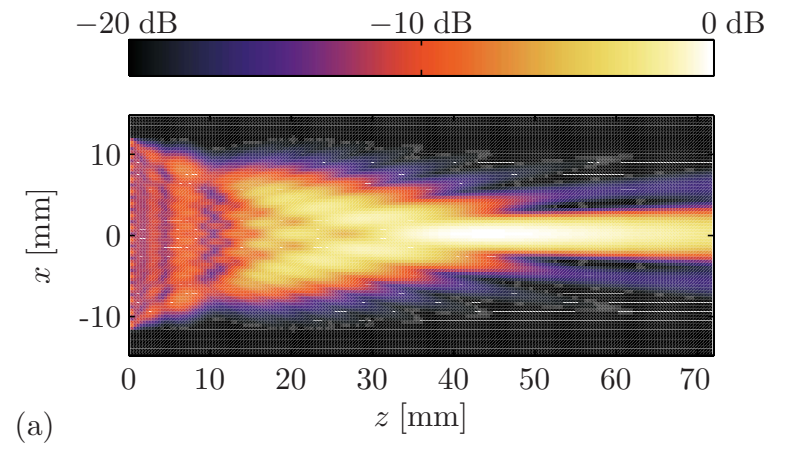

(a)

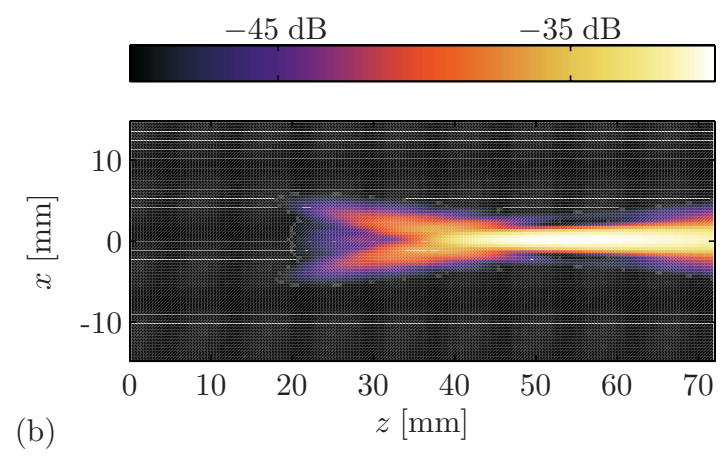

(b)
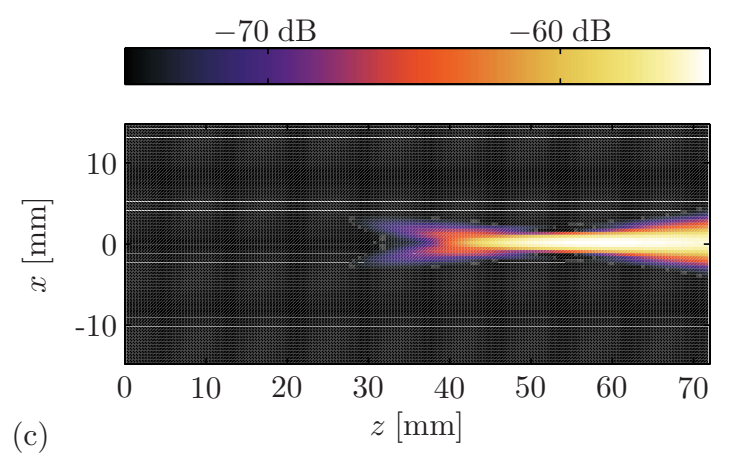

FIG. 9. (Color online) Spectral profiles in the plane $y=0 \mathrm{~mm}$ for the phased array transducer exciting an unsteered beam. (a) Fundamental spectral profile. (b) Second harmonic spectral profile. (c) Third harmonic spectral profile. The $0 \mathrm{~dB}$ level corresponds to $414 \mathrm{kPa}$.

tween both methods is observed. At the main beam, i.e., at $x=40 \mathrm{~mm}$, the level of the second harmonic component predicted by the AS-NLP method is $2.6 \mathrm{~dB}$ lower than the level predicted by the INCS method, and this difference increases for larger $x$. The third harmonic components obtained by both methods differ by $7.5 \mathrm{~dB}$ at the main beam, and the tails have a widely different shape and level. The case of steering off axis reveals two points. First, that the INCS method is capable of dealing with this type of fields, and second, that the quasi-planar wave assumption in the nonlinear step of the AS-NLP method yields a result of limited accuracy for fields that are not propagating in the assumed direction, i.e., perpendicular to the computational planes.

\section{DISCUSSION}

Since the INCS method is designed to be a directionally independent method, its discretization in all dimensions is coupled to the highest frequency of interest $F$. The presented comparisons confirm that the INCS method reproduces the nonlinear acoustic wavefield very accurately up to the limit
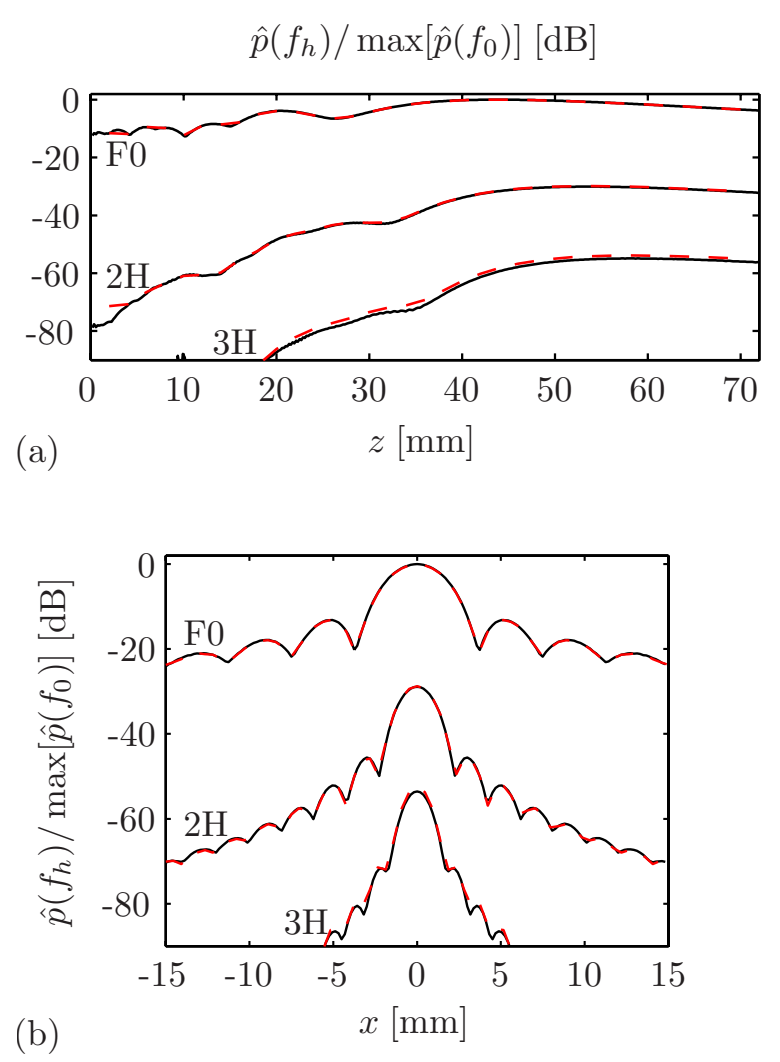

FIG. 10. (Color online) Spectral profiles for the phased array transducer exciting an unsteered beam, as obtained by the INCS method (solid) and by the AS-NLP method (dashed). (a) Axial spectral profiles. (b) Lateral spectral profiles at $y=0 \mathrm{~mm}$ and $z=58 \mathrm{~mm}$. The $0 \mathrm{~dB}$ level corresponds to $414 \mathrm{kPa}$.

$D_{F}=2$. To obtain accurate results for the $h$ th harmonic frequency component at a discretization $D_{F}=2$, it is found sufficient to take $F=(h+1) f_{0}$, and detailed investigation by the authors showed that even $F=(h+0.5) f_{0}$ showed good results. With regard to the number of iterations, it is found that for weak to moderate nonlinear distortion, the iteration $j=h+1$ already provides sufficient accuracy.

The diffraction and the nonlinear distortion are included without any dependence on the direction, which makes the INCS method fit for an arbitrarily directed, focused, or wideangle nonlinear acoustic wavefield, including sidelobes and grating lobes. The directional independence of the nonlinear distortion is particularly important for the accurate prediction of the higher harmonics of a steered beam. This is clearly illustrated by the comparison between the INCS method and the AS-NLP method in Fig. 12. An extra advantage of the method is that the utilized convolution method gives no reflections on the computational grid boundaries, as occurs for finite difference or finite element methods, and it has no periodical boundary conditions, which are an issue with pseudospectral methods.

Because the FFT method is utilized in the evaluation of the convolution sum, the amount of arithmetical operations required by the scheme is of order $N \log N$, where $N$ is the total number of grid points. The method can therefore be considered as near-linear in its computation time. Because of the coarse discretization at the limit $D_{F}=2$ in all dimensions, the INCS method is very efficient in terms of computation time and memory usage. Nevertheless, compared to forward- 

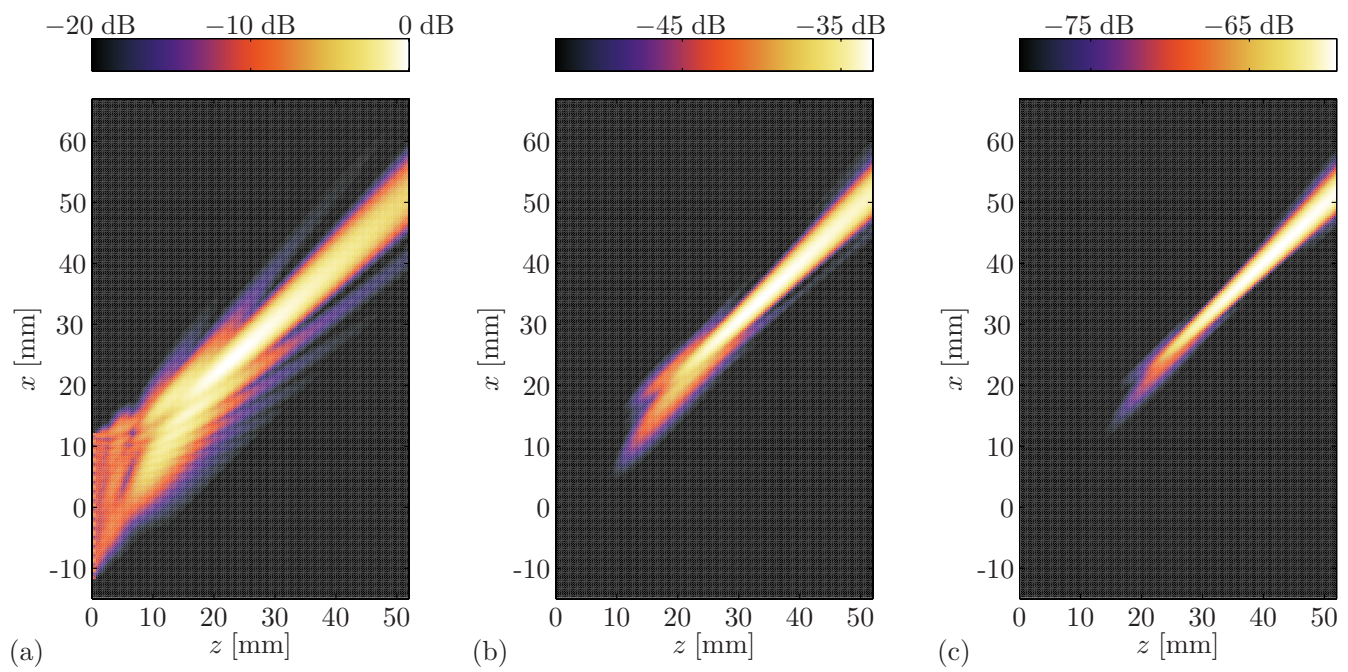

FIG. 11. (Color online) Spectral profiles in the plane $y=0 \mathrm{~mm}$ for the phased array transducer exciting a beam steered at $45^{\circ}$. (a) Fundamental spectral profile. (b) Second harmonic spectral profile. (c) Third harmonic spectral profile. The $0 \mathrm{~dB}$ level corresponds to $358 \mathrm{kPa}$.

wave methods such as the AS-NLP method or KZK-based methods, and compared to full-wave schemes with a marching-on-in-time scheme, an apparent disadvantage of the employed iterative scheme is the necessity to store the field data at the entire four-dimensional grid. This requires a computer with considerable memory, or a significant usage of the harddisk as backup storage, or alternatively the use of a distributed computer system. The simulations of the fields of the point source, the rectangular source, and the cylindrical source were performed on a desktop computer running at $1 \mathrm{GHz}$ and with $2 \mathrm{~GB}$ memory, and the execution times varied from $9 \mathrm{~min}$ to as much as $130 \mathrm{~h}$. However, the large execution time was caused mainly by disk access time. The computations of the nonlinear field from the phased array were performed on 16 processors of an SGI Altix 3700 clustered multiprocessor system consisting of 416 Intel Itanium processors running at $1.3 \mathrm{GHz}$ and with $2 \mathrm{~GB}$ memory per core. These simulations took between 1 and $3 \mathrm{~h}$.

\section{CONCLUSIONS}

In this paper, a novel, directionally independent method has been presented that predicts the three-dimensional, nonlinear acoustic pressure field from extended sources that ex-

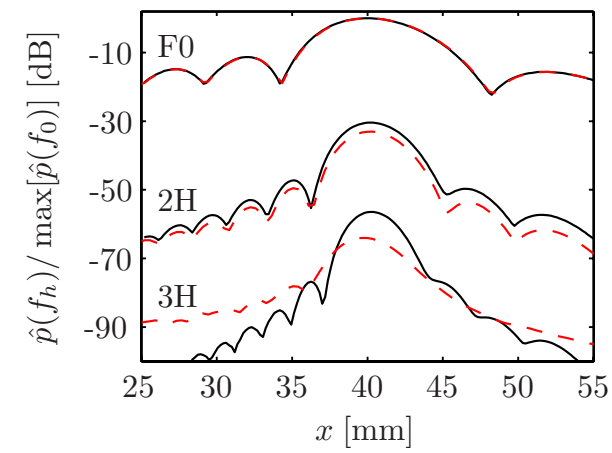

FIG. 12. (Color online) Lateral spectral profiles at $y=0 \mathrm{~mm}$ and $z$ $=40 \mathrm{~mm}$ for the phased array transducer exciting a beam steered at $45^{\circ}$, as obtained by the INCS method (solid) and by the AS-NLP method (dashed). The $0 \mathrm{~dB}$ level corresponds to $358 \mathrm{kPa}$. cite a pulsed wavefield propagating under an arbitrarily wide angle. The method is based on the Neumann iterative solution of the lossless Westervelt equation, where the nonlinear term is interpreted as a nonlinear contrast source. In the iterative scheme, the most demanding step in each iteration is the spatiotemporal convolution of Green's function with the primary source or with the nonlinear contrast source. With the employment of the filtered convolution method, ${ }^{26}$ this convolution may be performed accurately at a discretization of $D_{F}=2$ grid points per wavelength and per period at the maximum frequency of interest $F$. This enables the computation of acoustic wavefields over very large domains. The presented numerical results support the conclusion that the INCS method is directionally independent and produces very accurate results for the nonlinear acoustic wavefield in the case of weak to moderate nonlinearity, as occurs in medical diagnostic applications.

\section{ACKNOWLEDGMENTS}

This work was supported by the Dutch Technology Foundation (STW) and by the Dutch National Computing Facilities Foundation (NCF). The authors would like to thank Ayache Bouakaz, Richard Cobbold, and Roger Zemp for providing the source code of the comparison methods.

\section{APPENDIX A: DERIVATION OF THE NONLINEAR CONSTITUTIVE PARAMETERS FROM THE EQUATION OF STATE}

Often, the nonlinear behavior of the medium is derived from a second-order approximation of the equation of state relating the hydrostatic pressure $P$ to the mass density $\rho$ and the entropy $s$. Under the condition of isentropy, $P$ may then be written $\mathrm{as}^{5}$

$$
P=p_{0}+c_{0}^{2}\left(\rho-\rho_{0}\right)+\frac{c_{0}^{2}}{\rho_{0}} \frac{B}{2 A}\left(\rho-\rho_{0}\right)^{2},
$$

where $p_{0}$ is the ambient pressure, $\rho_{0}$ is the ambient mass density, $c_{0}$ is the small-signal sound speed, and $B / A$ is the 
parameter of nonlinearity. Since in the nonviscous case $P$ is related to the acoustic pressure by $p=P-p_{0}$, we can use the equation of state to write $\rho$ as a function of $p$, giving

$$
\rho=\rho_{0}+\rho_{0} \frac{A}{B}\left[\left(1+2 \frac{B}{A} \kappa_{0} p\right)^{1 / 2}-1\right],
$$

and to first-order approximation this becomes ${ }^{38}$

$$
\rho=\rho_{0}\left[1+\kappa_{0} p\right],
$$

where $\kappa_{0}=1 / \rho_{0} c_{0}^{2}$ is the ambient compressibility.

In order to arrive at an expression for $\kappa$, we combine the sourceless version of Eq. (2), i.e.,

$$
\boldsymbol{\nabla} \cdot \boldsymbol{v}+\kappa D_{t} p=0,
$$

and the conservation law of mass

$$
D_{t} \rho+\rho \boldsymbol{\nabla} \cdot \boldsymbol{v}=0,
$$

to obtain

$$
\kappa D_{t} p=\frac{D_{t} \rho}{\rho} .
$$

With $D_{t} \rho=(\mathrm{d} \rho / \mathrm{d} p) D_{t} p$ this leads to

$$
\kappa=\frac{1}{\rho} \frac{\mathrm{d} \rho}{\mathrm{d} p} .
$$

Upon substitution of Eq. (A2) for $\rho$ we end up with an expression for $\kappa(p)$, from which we can easily derive the firstorder approximation ${ }^{38}$

$$
\kappa=\kappa_{0}\left[1+\kappa_{0}(1-2 \beta) p\right],
$$

where $\beta=1+B / 2 A$ is the coefficient of nonlinearity.

\section{APPENDIX B: DERIVATION OF THE LOSSLESS WESTERVELT EQUATION FROM THE FIRST-ORDER ACOUSTIC EQUATIONS}

Substitution of the constitutive parameters of Eqs. (3) and (4) into field equations (1) and (2), and discarding the terms that involve third and higher order products of $p$ and/or $\boldsymbol{v}$ result in the set of equations

$$
\begin{gathered}
\boldsymbol{\nabla} p+\rho_{0} \partial_{t} \boldsymbol{v}=\boldsymbol{f}-\rho_{0} \kappa_{0} p \partial_{t} \boldsymbol{v}-\rho_{0}(\boldsymbol{v} \cdot \boldsymbol{\nabla}) \boldsymbol{v}, \\
\boldsymbol{\nabla} \cdot \boldsymbol{v}+\kappa_{0} \partial_{t} p=q-\kappa_{0}^{2}(1-2 \beta) p \partial_{t} p-\kappa_{0} \boldsymbol{v} \cdot \boldsymbol{\nabla} p .
\end{gathered}
$$

For a rotation free field the substitution $\boldsymbol{\nabla} \boldsymbol{v}=(\boldsymbol{\nabla v})^{T}$ is valid, and upon employment of the first-order approximation $\boldsymbol{\nabla} p=-\rho_{0} \partial_{t} \boldsymbol{v}$ in the second-order terms, and upon subsequent reordering of the terms, a new set of equations may be obtained as

$$
\begin{aligned}
\nabla p+\rho_{0} \partial_{t} \boldsymbol{v} & =\boldsymbol{f}-\boldsymbol{\nabla} L \\
\boldsymbol{\nabla} \cdot \boldsymbol{v}+\kappa_{0} \partial_{t} p & =q+\kappa_{0}^{2} \beta \partial_{t}^{2} p^{2}+\kappa_{0} \partial_{t} L,
\end{aligned}
$$

where

$$
L=\frac{1}{2} \rho_{0} \boldsymbol{v} \cdot \boldsymbol{v}-\frac{1}{2} \kappa_{0} p^{2}
$$

is the Lagrangian density. The second-order wave equation is obtained by subtracting the divergence of Eq. (B3) from $\rho_{0}$ times the temporal derivative of Eq. (B4). This directly leads to the source term $S=\rho_{0} \partial_{t} q-\nabla \cdot f$ in Eq. (5). Except for the source terms, Eqs. (B3)-(B5) are equivalent to the lossless versions of Eqs. (5)-(8) of Aanonsen et al., ${ }^{25}$ who showed that these equations lead to the Westervelt equation upon neglecting the local nonlinear effects that are represented by the terms involving $L$.

${ }^{1}$ B. Ward, A. C. Baker, and V. F. Humphrey, "Nonlinear propagation applied to the improvement of resolution in diagnostic medical ultrasound equipment," J. Acoust. Soc. Am. 101, 143-154 (1997).

${ }^{2}$ F. Tranquart, N. Grenier, V. Eder, and L. Pourcelot, "Clinical use of ultrasound tissue harmonic imaging," Ultrasound Med. Biol. 25, 889-894 (1999).

${ }^{3}$ A. Bouakaz and N. de Jong, "Native tissue imaging at superharmonic frequencies," IEEE Trans. Ultrason. Ferroelectr. Freq. Control 50, 496506 (2003).

${ }^{4}$ A. Bouakaz, E. Merks, C. T. Lancée, and N. Bom, "Noninvasive bladder volume measurements based on nonlinear wave distortion," Ultrasound Med. Biol. 30, 469-476 (2004).

${ }^{5}$ Nonlinear Acoustics, edited by M. F. Hamilton and D. T. Blackstock (Academic, San Diego, CA, 1998).

${ }^{6}$ K.-E. Frøysa, J. Naze Tjøtta, and S. Tjøtta, "Linear propagation of a pulsed sound beam from a plane or focusing source," J. Acoust. Soc. Am. 93, 80-92 (1993).

${ }^{7}$ M. D. Cahill and A. C. Baker, "Numerical simulation of the acoustic field of a phased-array medical ultrasound scanner," J. Acoust. Soc. Am. 104, 1274-1283 (1998).

${ }^{8}$ A. Bouakaz, C. T. Lancée, P. Frinking, and N. de Jong, "Simulations and measurements of nonlinear pressure field generated by linear array transducers," in Proceedings of the 1999 IEEE Ultrasonics (1999), pp. 15111514.

${ }^{9}$ V. A. Khokhlova, A. E. Ponomarev, M. A. Averkiou, and L. A. Crum, "Nonlinear pulsed ultrasound beams radiated by rectangular focused diagnostic transducers," Acoust. Phys. 52, 481-489 (2006).

${ }^{10} \mathrm{X}$. Yang and R. Cleveland, "Time domain simulation of nonlinear acoustic beams generated by rectangular pistons with application to harmonic imaging," J. Acoust. Soc. Am. 117, 113-123 (2005).

${ }^{11} \mathrm{P}$. T. Christopher and K. J. Parker, "New approaches to nonlinear diffractive field propagation," J. Acoust. Soc. Am. 90, 488-499 (1991).

${ }^{12}$ J. Tavakkoli, D. Cathignol, and R. Souchon, "Modeling of pulsed finiteamplitude focused sound beams in time domain," J. Acoust. Soc. Am. 104, 2061-2072 (1998).

${ }^{13}$ R. J. Zemp, J. Tavakkoli, and R. S. C. Cobbold, "Modeling of nonlinear ultrasound propagation in tissue from array transducers," J. Acoust. Soc. Am. 113, 139-152 (2003)

${ }^{14}$ J. Wojcik, A. Nowicki, P. A. Lewin, P. E. Bloomfield, T. Kujawska, and L. Filipczynski, "Wave envelopes method for description of nonlinear acoustic wave propagation," Ultrasonics 44, 310-329 (2006).

${ }^{15}$ T. Varslot and G. Taraldsen, "Computer simulation of forward wave propagation in tissue," IEEE Trans. Ultrason. Ferroelectr. Freq. Control 52, 1473-1482 (2005).

${ }^{16}$ P. D. Fox, A. Bouakaz, and F. Tranquart, "Computation of steered nonlinear fields using offset KZK axes," in Proceedings of the IEEE Ultrasonics 2005 (2005), pp. 1984-1987.

${ }^{17}$ T. Christopher, "Algorithm for the nonlinear propagation of acoustic beams from phased arrays and nonplanar sources," IEEE Trans. Ultrason. Ferroelectr. Freq. Control 53, 2188-2192 (2006).

${ }^{18}$ T. Varslot, S.-E. Masoy, T. Johansen, and B. Angelsen, "Aberration in nonlinear acoustic wave propagation," IEEE Trans. Ultrason. Ferroelectr. Freq. Control 54, 470 (2007).

${ }^{19} \mathrm{~V}$. W. Sparrow and R. Raspet, "A numerical method for general finite amplitude wave propagation in two dimensions and its application to spark pulses," J. Acoust. Soc. Am. 90, 2683-2691 (1991).

${ }^{20} \mathrm{~T}$. Yano and Y. Inoue, "Strongly nonlinear waves and streaming in the near field of a circular piston," J. Acoust. Soc. Am. 99, 3353-3372 (1996).

${ }^{21}$ M. Liebler, S. Ginter, T. Dreyer, and R. E. Riedlinger, "Full wave modeling of therapeutic ultrasound: Efficient time-domain implementation of the frequency power-law attenuation," J. Acoust. Soc. Am. 116, 27422750 (2004).

${ }^{22}$ I. M. Hallaj and R. O. Cleveland, "FDTD simulation of finite-amplitude pressure and temperature fields for biomedical ultrasound," J. Acoust. Soc. Am. 105, L7-L12 (1999).

${ }^{23}$ J. Hoffelner, H. Landes, M. Kaltenbacher, and R. Lerch, "Finite element 
simulation of nonlinear wave propagation in thermoviscous fluids including dissipation," IEEE Trans. Ultrason. Ferroelectr. Freq. Control 48, 779786 (2001).

${ }^{24}$ G. Wojcik, J. Mould, L. Carcione, and S. Ayter, "A study of second harmonic generation by focused medical transducer pulses," in Proceedings of the 1998 IEEE Ultrasonics (1998), pp. 1583-1588.

${ }^{25}$ S. I. Aanonsen, T. Barkve, J. Naze Tjotta, and S. Tjotta, "Distortion and harmonic generation in the nearfield of a finite amplitude sound beam," J. Acoust. Soc. Am. 75, 749-768 (1984).

${ }^{26}$ M. D. Verweij and J. Huijssen, "A filtered convolution method for the computation of acoustic wave fields in very large spatiotemporal domains," J. Acoust. Soc. Am. 125, 1868-1878 (2009).

${ }^{27}$ A. T. de Hoop, Handbook of Radiation and Scattering of Waves (Academic, San Diego, CA, 1995)

${ }^{28}$ J. Huijssen, "Modeling of nonlinear medical diagnostic ultrasound," Ph.D. thesis, Delft University of Technology, Delft, The Netherlands (2008).

${ }^{29}$ J. T. Fokkema and P. M. van den Berg, Seismic Applications of Acoustic Reciprocity (Elsevier, Amsterdam, 1993).

${ }^{30}$ G. F. Roach, Green's Functions (Cambridge University Press, Cambridge, 1982).
${ }^{31}$ J. A. DeSanto, Scalar Wave Theory-Green's Functions and Applications (Springer-Verlag, Berlin, 1992).

${ }^{32}$ R. O. Cleveland, M. F. Hamilton, and D. T. Blackstock, "Time-domain modeling of finite-amplitude sound in relaxing fluids," J. Acoust. Soc. Am. 99, 3312-3318 (1996).

${ }^{33}$ M. Frigo and S. G. Johnson, "The design and implementation of FFTW3," Proc. IEEE 93, 216-231 (2005).

${ }^{34}$ B. Fornberg, A Practical Guide to Pseudospectral Methods (Cambridge University Press, New York, 1996).

${ }^{35}$ J. de Koning, "Parallelizing Parnac," Technical Report No. TR06-03, VORtech Computing, Delft, The Netherlands, 2007.

${ }^{36}$ J. Huijssen, A. Bouakaz, M. D. Verweij, and N. de Jong, "Simulations of the nonlinear acoustic pressure field without using the parabolic approximation," in Proceedings of the IEEE Ultrasonics 2003 (2003), pp. 18511854.

${ }^{37}$ Y.-S. Lee and M. F. Hamilton, "Time-domain modeling of pulsed finiteamplitude sound beams," J. Acoust. Soc. Am. 97, 906-917 (1995).

${ }^{38}$ J. Huijssen and M. D. Verweij, "Nonlinear constitutive equations derived for fluids obeying an ideal gas, a Tait-Kirkwood or a B/A type equation of state," in Proceedings of the ISNA17 (2005), pp. 552-555. 